\title{
Réjean PELLETIER
}

Professeur, science politique, Université Laval

(1980)

\section{"Les partis}

\section{politiques et l'État."}

Un document produit en version numérique par Jean-Marie Tremblay, bénévole, professeur de sociologie au Cégep de Chicoutimi

Courriel: jean-marie tremblay@uqac.ca

Site web pédagogique : http://www.uqac.ca/jmt-sociologue/

Dans le cadre de: "Les classiques des sciences sociales" Une bibliothèque numérique fondée et dirigée par Jean-Marie Tremblay, professeur de sociologie au Cégep de Chicoutimi

Site web: http://classiques.uqac.ca/

Une collection développée en collaboration avec la Bibliothèque Paul-Émile-Boulet de l'Université du Québec à Chicoutimi

Site web: http://bibliotheque.uqac.ca/ 


\section{Politique d'utilisation de la bibliothèque des Classiques}

Toute reproduction et rediffusion de nos fichiers est interdite, même avec la mention de leur provenance, sans l'autorisation formelle, écrite, du fondateur des Classiques des sciences sociales, Jean-Marie Tremblay, sociologue.

Les fichiers des Classiques des sciences sociales ne peuvent sans autorisation formelle:

- être hébergés (en fichier ou page web, en totalité ou en partie) sur un serveur autre que celui des Classiques.

- servir de base de travail à un autre fichier modifié ensuite par tout autre moyen (couleur, police, mise en page, extraits, support, etc...),

Les fichiers (.html, .doc, .pdf., .rtf, .jpg, .gif) disponibles sur le site Les Classiques des sciences sociales sont la propriété des Classiques des sciences sociales, un organisme à but non lucratif composé exclusivement de bénévoles.

Ils sont disponibles pour une utilisation intellectuelle et personnelle et, en aucun cas, commerciale. Toute utilisation à des fins commerciales des fichiers sur ce site est strictement interdite et toute rediffusion est également strictement interdite.

\section{L'accès à notre travail est libre et gratuit à tous les utilisa- teurs. C'est notre mission.}

Jean-Marie Tremblay, sociologue

Fondateur et Président-directeur général,

LES CLASSIQUES DES SCIENCES SOCIALES. 
Cette édition électronique a été réalisée par Jean-Marie Tremblay, bénévole, professeur de sociologie au Cégep de Chicoutimi à partir de :

\section{Réjean PELLETIER}

\section{"Les partis politiques et l'État."}

Un texte publié dans l'ouvrage sous la direction de Gérard Bergeron et Vincent Lemieux, L'État du Québec en devenir, chapitre 8, pp. 241-261. Montréal : Les Éditions du Boréal Express, 1980, 413 pp.

[Autorisation formelle accordée par l'auteur le 18 octobre 2004 de diffuser ce texte dans Les Classiques des sciences sociales.]

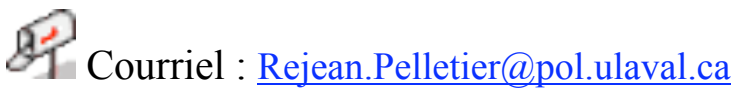

Polices de caractères utilisée :

Pour le texte: Times New Roman, 12 points.

Pour les citations : Times New Roman, 12 points.

Pour les notes de bas de page : Times New Roman, 12 points.

Édition électronique réalisée avec le traitement de textes Microsoft Word 2008 pour Macintosh.

Mise en page sur papier format : LETTRE (US letter), 8.5', x 11'’)

Édition numérique réalisée le $1^{\text {er }}$ juin 2009 à Chicoutimi, Ville de Saguenay, province de Québec, Canada.

\section{Fait avec




\section{Réjean PELLETIER}

Professeur, science politique, Université Laval

\section{“Les partis politiques et l’État.”}

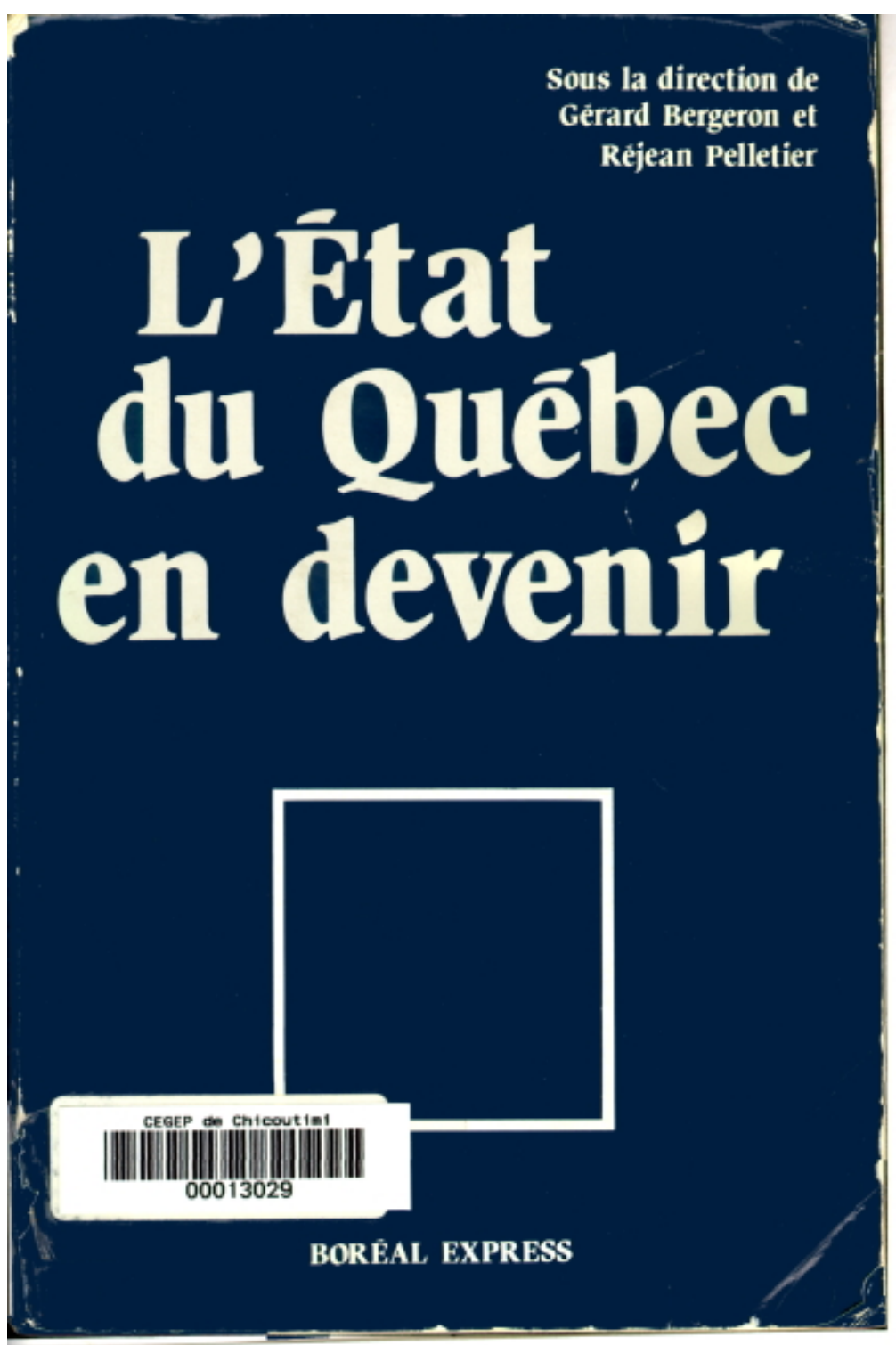

Un texte publié dans l'ouvrage sous la direction de Gérard Bergeron et Vincent Lemieux, L'État du Québec en devenir, chapitre 8, pp. 241-261. Montréal : Les Éditions du Boréal Express, 1980, 413 pp. 


\section{Table des matières}

$\underline{\text { Introduction }}$

La rupture de 1960

Les partis politiques et l'interventionnisme étatique (1960-1968)

La rupture de 1968

Les partis et le fédéralisme canadien, 1968-1976

Conclusion

Figure 1. Opposition globale du PLQ et de l'UN sur deux axes, 1960-1968

Figure 2. Position globale des partis politiques québécois

Figure 3. Position globale des partis politiques québécois sur deux axes en 1970 


\section{Réjean PELLETIER}

\section{“Les partis politiques et l'État”. *}

Un texte publié dans l'ouvrage sous la direction de Gérard Bergeron et Vincent Lemieux, L'État du Québec en devenir, chapitre 8, pp. 241-261. Montréal : Les Éditions du Boréal Express, 1980, 413 pp.

\section{Introduction}

\section{$\underline{\text { Retour à la table des matières }}$}

Tenter d'analyser les partis politiques du Québec au cours de la période 19601976, c'est inévitablement faire référence aux transformations mêmes de la société québécoise, à ses problèmes et à son évolution, aux nouvelles idéologies dominantes et à la transformation du pouvoir ${ }^{1}$. Pour cerner cette relation dialectique entre les partis et la société, on peut privilégier deux voies complémentaires : l'analyse des programmes et l'analyse des politiques.

La première situe plutôt les partis au niveau sociétal ou comme points de jonction entre le social et le politique puisque les partis, dans leurs programmes, identifient des problèmes qui confrontent la société, formulent des solutions pour résoudre ces problèmes et concrétiser l'action du parti, et définissent des objectifs et des finalités qui peuvent orienter le développement de cette société ${ }^{2}$. En d'autres

* Je tiens à remercier tous les étudiants qui ont participé à mon séminaire de 2e et 3e cycles sur les partis politiques et, en particulier, mon assistante de recherche, Paule Duchesneau, qui a épluché patiemment les programmes des partis et qui a contribué à l'orientation de la recherche.

1 Pour une étude générale sur les partis, voir entre autres Jean Charlot, Les partis politiques. A. Colin, « Dossier U », 1971.

2 Cette définition est empruntée à Daniel Latouche, « Le contenu thématique et l'orientation idéologique des programmes électoraux en 1973 » dans Daniel 
termes, la perception des problèmes et la définition des moyens reposent sur une certaine conception de la société qui devrait normalement transparaître dans l'analyse des programmes.

L'analyse des politiques situe plutôt les partis comme points de jonction entre le politique et le social puisque, au pouvoir, les partis cherchent habituellement à mettre en oeuvre leur programme ${ }^{3}$ de façon à résoudre certains problèmes sociaux par des politiques de distribution, de régulation ou de redistribution ${ }^{4}$. Ce qui traduit aussi une conception particulière de l'État, soit comme distributeur ou redistributeur de biens et de services, soit comme créateur de structures et d'instruments d'action, soit tout simplement comme ordonnateur des choses et des hommes 5 .

Dans le cadre restreint de cet article, seule la première voie sera explorée. Il s'agira donc essentiellement de considérer les partis comme agents de transformation sociale à travers l'analyse de leurs programmes et de certains documents importants, de façon à dégager le mieux possible à la fois leur volonté d'action et leur conception globale de la société québécoise au cours de la période 19601976.

Mais il importe aussi de souligner que si dans certains partis, comme le Parti québécois par exemple, le programme est surtout l'œuvre de militants qui participent à son élaboration et qui tentent d'en définir les orientations idéologiques fondamentales, il en va autrement dans d'autres partis. Ainsi, le programme du Parti libéral en 1960 fut l'œuvre exclusive d'un petit groupe de dirigeants, réunis autour du chef. D'autres programmes peuvent provenir à la fois d'un groupe d'experts

Latouche, G. Lord et Jean-Guy Vaillancourt, dir., Le processus électoral au Québec: les élections provinciales de 1970 et 1973, Montréal, Hurtubise HMH, 1976, p. 128.

3 Contrairement à la croyance populaire qui soutient que, au pouvoir, les partis oublient rapidement leurs programmes. Depuis 1960 au Québec, les programmes ont été une source importante d'inspiration des politiques conçues par les différents gouvernements.

4 Theodore J. Lowi, «American Business, Public Policy, Case-Studies, and Political Theory », World Politics, 16 : 4, juillet 1964, pp. 677-715 et surtout p. 689-691.

5 Voir Vincent Lemieux, "Quel État du Québec ? », Études internationales, $13: 2$, juin 1977, p. 260. 
recrutés à l'extérieur du parti et des militants eux-mêmes : c'est le cas du programme de l'Union nationale en 1966, qui fut rédigé par un comité restreint, secondé par quelques groupes de travail, mais qui était basé sur les résolutions votées aux assises de mars 1965.

Cependant, dans tous ces cas, le programme est présenté comme un document officiel du parti qui traduit ses grandes orientations idéologiques et une certaine conception de la société québécoise. De là l'importance d'analyser de tels documents qui situent les partis au point de jonction du social et du politique.

\section{La rupture de 19606}

$\underline{\text { Retour à la table des matières }}$

Au Québec, en 1960, l'urbanisation et l'industrialisation sont des faits déjà acquis et la décennie qui s'ouvre alors marque, a ce point de vue, une continuité et non une rupture avec la période antérieure (1945-1960).

En effet, la population du Québec est déjà majoritairement urbaine dès 1921 selon les définitions du recensement canadien - et ce phénomène ne fait que s'amplifier d'une façon régulière dans les décennies suivantes, si l'on excepte la période de la grande crise économique mondiale des années trente.

Au niveau économique, on observe aussi un certain nombre de changements structurels importants. À partir de la Seconde Guerre mondiale, chacun des grands secteurs d'activité économique subit des mutations qui affectent globalement la société québécoise. On constate, en effet, un déclin constant et prononcé de la main-d'œuvre occupée dans le secteur primaire ${ }^{7}$. Par contre, le secteur secondaire (défini comme le secteur de la fabrication ou le secteur manufacturier) croît constamment jusqu'en 1951 pour ensuite décliner et atteindre en 1971 un pourcen-

6 Les principaux éléments de cette section sont déjà parus, sous une forme plus élaborée que celle-ci, dans un article intitulé " Nationalisme et étatisme au Québec dans les années 60 : une hypothèse de travail pour l'analyse des programmes des partis politiques ", Revue canadienne des études sur le nationalisme, $7: 2$, automne 1980.

7 Le secteur primaire regroupe les secteurs agricole, minier et forestier. Mais c'est surtout dans le secteur agricole que ce déclin s'est fait sentir. 
tage de main-d'œuvre inférieur à celui de 1941. Le déclin de ces deux secteurs s'effectue au profit du secteur tertiaire, qui se développe considérablement au cours des années cinquante, surtout dans le tertiaire privé, et qui continue à croître au cours de la décennie suivante par suite de la montée rapide du tertiaire public.

Le développement du secteur tertiaire après la Seconde Guerre mondiale est relié à la croissance des grandes villes et, en particulier, de la région métropolitaine de Montréal. Dans ces milieux urbanisés, en même temps que se développent les secteurs du commerce, de la finance, des assurances et des communications, il devient aussi nécessaire de créer des institutions d'éducation, de santé et de bienêtre qui sont gérées par des administrateurs ayant les compétences nécessaires et choisis de plus en plus, non pas parmi les rangs du clergé qui ne peut suffire seul à la tâche, mais parmi les jeunes diplômés des sciences sociales et administratives. C'est ainsi que se forme et croît, par suite de changements structurels importants, une nouvelle classe moyenne, que le sociologue Hubert Guindon définit comme étant le « produit de l'expansion bureaucratique des organisations ${ }^{8}$ ».

Dans cette optique, la rupture de 1960 se situe avant tout au niveau politique plutôt que socio-économique, puisque les changements structurels importants sont déjà en marche depuis la Seconde Guerre mondiale. Cette rupture se caractérise certes par l'arrivée au pouvoir d'un autre parti politique, mais ce parti se fonde surtout sur la nouvelle classe moyenne ou, plus précisément, sur une nouvelle élite dirigeante issue de cette classe. C'est donc par le biais du Parti libéral que cette élite va en grande partie se manifester, réaliser ses réformes socioéconomiques et affirmer son néo-nationalisme.

8 Hubert Guindon, «Social Unrest, Social Class and Quebec's Bureaucratic Revolution », Queen's Quarterly, $71: 7$, été 1974, p. 152. Mais il faut aussi souligner que la nouvelle classe moyenne ne recouvre pas l'ensemble du secteur tertiaire - elle exclut à la fois la plupart des notables de province et les employés subalternes du secteur des services pour englober essentiellement des personnes travaillant à un niveau supérieur du secteur tertiaire : hauts fonctionnaires des administrations publiques, cadres et directeurs des services de santé et d'enseignement, des organisations syndicales et coopératives et des média d'information, ainsi que des universitaires. [Voir la version française de cet article sous le titre: "Réexamen de l'évolution sociale du Québec" dans Les Classiques des sciences sociales. JMT.] 
Cependant, le Parti libéral ne peut résumer à lui seul la rupture de 1960 : il « exprime l'esprit nouveau » de cette époque, comme l'écrivait l'historien Ramsay Cook, et « manifeste la volonté d'en inspirer sa politique 9 », mais il n'apparaît pas comme l'unique créateur de cet esprit nouveau, bien qu'il en ait été l'un des principaux promoteurs. Il apparaît plutôt à la fois comme un moteur et un instrument de cette rupture en servant de moyen d'expression au néo-nationalisme et de moyen d'action pour le mettre en oeuvre.

La nouvelle classe moyenne s'était trouvée confrontée dans les années cinquante à trois « institutions » de blocage qui occupaient tout le champ sociétal : l'Église catholique, qui contrôlait en grande partie le domaine des affaires sociales et de l'éducation; la bourgeoisie anglophone (canadienne et continentale), qui dominait le secteur économique et enfin le gouvernement fédéral, qui s'imposait largement dans le secteur politique par suite de la centralisation des pouvoirs et des ressources à Ottawa et par suite du rejet du gouvernement duplessiste par une bonne partie de la nouvelle classe moyenne.

Pour contrer l'action de ces «institutions », la nouvelle classe moyenne qui accède au pouvoir en 1960 va se servir de l'État québécois comme instrument d'action collective et définir une nouvelle idéologie, le néo-nationalisme, qui viendra en même temps justifier son action. Cela explique les interventions de l'État à la fois dans les secteurs d'activité contrôlés traditionnellement par l'Église, dans le domaine économique, par la création d'entreprises publiques gérées par des francophones, et dans le champ politique, en s'opposant à la présence de l'État fédéral dans certains secteurs.

Ainsi, la rupture de 1960 implique tout à la fois une action motrice de l'État, des interventions étatiques dans de nouveaux secteurs d'activité et le passage d'un nationalisme à dominante culturelle axé sur la langue et la foi à un nationalisme à dominante politique axé sur l'État, qui devient la nouvelle « institution » dominante à la place de l'Église.

C'est ce rôle capital dévolu à l'État dans la vie économique, sociale et culturelle qui définit essentiellement le néo-nationalisme, par opposition au nationalisme conservateur traditionnel, qui sécrétait une profonde méfiance à l'égard de l'État.

9 Ramsay Cook, Le Sphinx parle français, Montréal, HMH, coll. « Aujourd'hui », 1968, p. 23. 
Toutes les autres caractéristiques en découlent : c'est parce qu'il est étatiste que le néo-nationalisme est revendicatif et offensif et non plus défensif, politique et non plus seulement culturel, de plus en plus souverainiste et non plus seulement autonomiste, québécois et non plus canadien-français, rattaché à des valeurs technocratiques et non plus religieuses et ruralistes.

C'est ce même néo-nationalisme étatiste - et c'est là mon hypothèse centrale qui a inspiré (ou non, selon le cas) les programmes et l'idéologie globale des partis politiques au Québec depuis les années soixante et qui permet de démarquer les partis les uns des autres. En d'autres termes, ce qui caractérise les partis politiques québécois au cours de la période 1960-1976, c'est essentiellement leur position face à l'utilisation et à l'intervention de l'État dans la société québécoise. À cet égard, on peut diviser cette période en deux, l'année 196810 constituant une année charnière dans la vie politique québécoise et canadienne.

\section{Les partis politiques et l'interventionnisme étatique (1960-1968)}

$\underline{\text { Retour à la table des matières }}$

Par interventionnisme, j'entends toute mesure favorisant la croissance de l'État ou du secteur public et parapublic (création de ministères ou de régies, nationalisations) ou toute forme de contrôle étatique sur un secteur d'activité. Je qualifierais de non-interventionniste, toute mesure qui favorise la non-croissance de l'État (refus d'intervention ou rétrécissement de son champ d'action) de même que toute mesure favorable au secteur prive, a son développement, à sa croissance (et cela peut prendre la forme de subventions) ou qui incite le secteur privé à faire telle ou telle chose 11 .

L'intervention étatique peut prendre une double orientation : une orientation individualiste, par des mesures de distribution ou de redistribution individuelle

10 Voir infra, « La rupture de 1968 ».

11 Il importe de préciser que le non-interventionnisme ainsi défini n'est pas le simple équivalent du laisser-faire en matières économiques. 
impliquant prestation directe de biens et services aux individus, même si ces mesures ont un caractère universel (c'est-à-dire touchent tous les individus) ; une orientation collectiviste, par des mesures favorables à la collectivité sans prestation directe de biens et services (nationalisations, politiques linguistiques, etc. ${ }^{12}$ ).

Ce qui caractérise les partis politiques du Québec au cours de la période 19601968 et ce qui les démarque les uns des autres, c'est essentiellement leur position sur l'axe interventionniste. Dans l'ensemble, le Parti libéral apparaît nettement plus interventionniste que l'Union nationale, surtout jusqu'à ce que celle-ci tienne son congrès d'orientation de mars 1965 et élabore son programme politique de $1966^{13}$.

Lorsqu'on analyse les programmes du Parti libéral de 1960 et de 1966, on constate une accentuation - du moins en termes quantitatifs - du rôle que doit jouer l'État québécois (augmentation de 79 à 91 propositions interventionnistes et diminution de 19 à 11 propositions seulement qui se situent au pôle non interventionniste). On assiste en même temps à une réorientation des priorités ${ }^{14}$ puisque, dans le programme de 1960, l'action étatique était concentrée d'abord dans les domaines politique et social et ensuite dans les domaines économique et culturel. En mettant ainsi l'accent sur le politique, ce programme annonce déjà un État québécois à construire qui prendra forme par la création ou la réorganisation de ministères et de régies de toutes sortes et par la modernisation de la fonction pu-

12 Il est évident que les propositions d'un programme, soit sous forme d'engagement du parti, soit sous forme d'énoncé de principes, ne peuvent pas toutes être classées sur ces axes. Ainsi, pour ne prendre qu'un exemple dans le programme de 1960 du Parti libéral, l'article 40 (convocation par Québec d'une conférence interprovinciale) est une proposition non classée, mais comptabilisée dans l'ensemble du programme, alors que l'article 27 (institution immédiate d'un système gouvernemental d'assurance-hospitalisation) est classé comme une proposition interventionniste (croissance de l'État) et individualiste (prestation directe de biens et services à tous les individus).

13 D'ailleurs, même en 1966, le Parti libéral apparaît un peu plus interventionniste que l'Union nationale ( $57 \%$ des propositions de l'ensemble du programme contre 54\% pour l'Union nationale).

14 Toujours en termes quantitatifs. On dénombre, en 1960, 29 propositions interventionnistes dans le domaine politique, 23 dans le domaine social, 21 dans le domaine économique et 6 seulement dans le domaine culturel. En 1966, 32 dans le domaine social, 23 dans le domaine économique et 20 chacune pour les domaines culturel et politique. 
blique. En 1966, l'interventionnisme du Parti libéral se concentre d'abord dans les secteurs social et économique puis, à égalité, dans les secteurs culturel et politique, bien que ce soit le domaine culturel qui connaisse la croissance la plus importante depuis 1960 .

Ainsi, la période qui s'ouvre avec les élections de 1966 semble un peu moins propice à la création d'appareils étatiques (sauf dans le domaine économique ${ }^{15}$ ), alors que le Parti libéral ressent de plus en plus le besoin de légiférer en matière linguistique afin de donner un visage français au Québec et d'assurer la priorité à la langue française dans tous les secteurs d'activité. Cette volonté de légiférer dans le domaine linguistique annonce l'un des thèmes importants de la période suivante (1968-1976) qui sera marquée non seulement par les problèmes constitutionnels mais aussi par l'action de l'État québécois pour assurer la prédominance de la langue française.

En somme, si dès 1960 le Parti libéral veut assurer un développement planifié du Québec sous l'égide d'un secteur public plus fort et plus compétent et qu'il accorde à l'État un rôle moteur dans cette croissance, en 1966 cette situation est non seulement confirmée, mais aussi accentuée, surtout dans les secteurs économique et culturel. Désormais, la prospérité économique et l'épanouissement culturel du Québec ne semblent vraiment assurés que par une action significative de l'État qui se veut plus directif et contrôleur.

Cette vision de l'action étatique se trouve d'ailleurs confirmée par certaines déclarations des dirigeants du parti. Ainsi, dès juin 1961, Jean Lesage affirmait : « La conjoncture présente nous force a repenser nos positions traditionnelles. Il nous faut des moyens puissants, non seulement pour relever les défis inévitables que nous rencontrerons dans les années qui viennent, mais aussi pour mettre le peuple canadien-français au diapason du monde actuel. Or le seul moyen puissant que nous possédions, c'est l'État du Québec, c'est notre État. Nous ne pouvons pas nous payer le luxe de ne pas l'utiliser. Je n'ai pas le droit, comme premier ministre

15 En termes quantitatifs, la différence est minime entre 1960 et 1966 : le PLQ propose de créer ou de réorganiser 17 ministères, régies, offices ou commissions en 1960 contre 15 en 1966, mais par rapport à l'ensemble des propositions interventionnistes, la différence devient plus significative. 
du gouvernement de cet État, de vous dire qu'il faut nous en remettre en cette matière aux seuls efforts des individus ou des groupements organisés ${ }^{16}$. »

Cette conception du rôle de l'État considère comme puissant et principal levier d'action est réaffirmée avec force dans le préambule du programme de 1966 : « Le Parti libéral du Québec, y lit-on, croit que l'État québécois est l'instrument principal de l'émancipation et du progrès de notre peuple. C'est à lui de mettre en marche et de coordonner les indispensables politiques de développement économique et social ${ }^{17}$. »

Même si le programme de 1960 laisse entrevoir quelques relents de nationalisme traditionnel, déjà les principaux éléments d'un néo-nationalisme fortement étatique sont bien en place. Il ne s'agit évidemment pas d'utiliser, dans le discours politique, le mot ou l'idée de "nationalisme" encore identifié à des valeurs conservatrices et à l'anti-étatisme. Mais en recourant à l'État québécois et en réclamant la modernisation de la fonction publique, le Parti libéral commence déjà à définir un néo-nationalisme qui sera reconnu comme tel quelques années plus tard. C'est ainsi que dès l'élection de 1962, le parti se montre plus nettement néonationaliste en favorisant la nationalisation des compagnies d'électricité et surtout en présentant cette nationalisation comme la condition première de la libération économique du Québec, pour être enfin «maîtres chez nous ». Le programme de 1966 reste fidèle à cette orientation, puisqu'il place l'État québécois au cœur du développement économique et social et qu'il considère cet État comme un instrument d'émancipation et de progrès.

Par ailleurs, en 1960, l'Union nationale se présente devant l'électorat sans véritable programme ; seule la publicité électorale peut nous renseigner quelque peu sur ses orientations. Or cette publicité est axée avant tout sur les réalisations du

16 Discours prononcé le 3 juin 1961 à Ottawa devant la Fédération des sociétés Saint-Jean-Baptiste et reproduit en partie dans jean Lesage, Un Québec fort dans une nouvelle Confédération, Québec, 1965, p. 18.

17 Préambule de Québec en marche. Le programme politique du Parti libéral du Québec, 1966, p. 3. 
parti, si bien que, à l'ouverture de sa campagne, Antonio Barrette ne craint pas d'annoncer : « Mon programme, c'est celui de 1944 18. »

L'Union nationale s'engage à défendre l'autonomie provinciale face à la volonté de centralisation du gouvernement fédéral et préconise en même temps la politique de porte ouverte pour les investissements étrangers. C'est cette même politique d'aide et de maintien du secteur privé qui doit présider au développement du secteur des affaires sociales et de l'éducation, puisque le meilleur système est encore celui qui dépend le moins de l'État. Bref, une politique de continuité fondée sur les réalisations du parti et de fidélité au nationalisme traditionnel, qui inspirait une profonde méfiance à l'égard de l'action étatique et s'appuyait sur un autonomisme défensif face à Ottawa. À l'occasion, on fait timidement allusion aux mesures « progressistes » du premier ministre Sauvé.

En 1962, l'Union nationale présente un « programme » où le secteur économique occupe la place la plus importante (39\% des propositions). Le parti tente d'ajuster ses formules à l'actualité politique et adopte un discours plus nettement néo-nationaliste : on parle d'indépendance financière, de souveraineté politique, d'État du Québec, et on préconise une conception élargie du rôle de l'État dans le développement économique. Mais du même souffle on s'engage à « entourer d'un climat de confiance et de sécurité l'entreprise privée compétente et dynamique de façon à encourager l'expansion industrielle du Québec ${ }^{19}$. »

De même, l'Union nationale défend la conception d'un État bienfaiteur ou redistributeur des richesses dans le secteur social en s'adressant surtout aux marginaux et aux moins nantis, tout en tablant sur le respect des droits des parents et de l'Église dans le domaine de l'éducation. En somme, on s'aperçoit que le parti est en période de mutation et d'ajustement à une nouvelle conjoncture créée par l'arrivée au pouvoir du Parti libéral en 1960. L'Union nationale, en 1962, n'a pas encore renié son passé, mais commence à s'en détacher de plus en plus.

18 Cité par jean Hamelin et André Garon, « La vie politique au Québec de 1956 à 1966 » dans Vincent Lemieux (dir.), Quatre élections provinciales au Québec, 1956-1966, Québec, PUL, 1969, pp. 12-13.

19 Voir le programme de 1962 de l'Union nationale publié par Jean-Louis Roy, Les programmes électoraux du Québec, Montréal, Leméac, tome Il, 1971, pp. 395-402. 
Il faudra attendre le congrès d'orientation de mars 1965 et surtout le programme de 1966 pour connaître vraiment une «nouvelle » Union nationale. En termes quantitatifs, ce programme accorde la priorité au secteur politique (42\% des propositions), suivi des domaines économique, culturel et social. On y constate en outre une augmentation sensible du nombre de propositions interventionnistes (passant de 31\% de l'ensemble du programme en 1962 à 54\% en 1966) et une diminution du nombre de propositions non interventionnistes (de 8,5\% en 1962 à $5 \%$ en 1966).

L'interventionnisme de l'Union nationale se manifeste désormais tant dans le domaine politique qu'au chapitre de la culture et des loisirs. Au plan social, elle va parfois plus loin que le Parti libéral en offrant, pour ne citer qu'un exemple, la possibilité de faire la grève même pendant la durée d'une convention collective. C'est plutôt dans le domaine économique que ses politiques peuvent apparaître un peu moins interventionnistes que celles du Parti libéral, encore que dans le secteur forestier, elle préconise une intervention massive de l'État. Ainsi, le processus d'évolution qui avait été enclenché en 1962 se reflète de plus en plus dans le programme de 1966. L'Union nationale a épousé les thèses du néo-nationalisme dans la vie politique, sociale et culturelle. Dans le secteur économique, son programme apparaît plutôt comme un mélange de conservatisme et de progressisme.

Aux termes de cette brève analyse, il apparaît donc que ce qui oppose davantage le Parti libéral et l'Union nationale, c'est leur position face à l'interventionnisme étatique. En d'autres mots, le consensus idéologique apparent qui dominait la scène québécoise a été brisé en 1960 selon cette ligne de clivage opposant les interventionnistes aux non-interventionnistes, et ce sont les interventionnistes de la nouvelle classe moyenne qui l'ont emporté.

D'ailleurs, si l'on cherche à opposer ces deux partis sur l'axe fédéralismeindépendantisme, on constate rapidement que les deux ne remettent pas fondamentalement en cause l'option fédéraliste, bien qu'ils puissent s'objecter aux visées centralisatrices d'Ottawa, réclamer des sources de revenu additionnelles pour le Québec et chercher à redéfinir le régime constitutionnel. Parlant de la réorientation du régime fédéral, le premier ministre Lesage déclarait qu'elle exigeait avant tout « un changement d'attitude à l'égard de la place et du rôle de l'élément fran- 
çais au Canada 20 ». Devant les membres de la Fédération libérale, il ajoutait que le Québec, en tant qu'expression politique du Canada français, « doit posséder et contrôler, dans la mesure du possible, les leviers économiques, sociaux, administratifs et politiques grâce auxquels, et grâce auxquels seulement, il pourra réaliser ses aspirations légitimes de peuple adulte 21 ». S'il accepte le cadre fédéral, le parti n'en exige donc pas moins une réorientation dans le respect des compétences accordées à chacun des niveaux de gouvernement et la reconnaissance du caractère distinctif du Québec.

C'est globalement le même esprit qui sous-tend l'autonomisme de l'Union nationale fondé sur la défense des juridictions provinciales face aux empiétements d'Ottawa, même si cet autonomisme prend un caractère de plus en plus revendicatif et offensif. Certes, Daniel Johnson a déjà écrit qu'il était « prêt à sauver l'autonomie du Québec même au prix de la Confédération » et qu'il ne fallait pas rejeter a priori la « solution séparatiste 22 »; il ajoutait néanmoins qu'il préférait, avant de se résoudre à « l'ultime recours du séparatisme », tenter tout ce qui peut encore être tenté pour que «la nation canadienne-française puisse se sentir chez elle, comme dans une véritable patrie, dans la totalité du Canada 23 ». C'est avant tout l'égalité qu'il recherchait, par un renouvellement du fédéralisme, plutôt que l'indépendance.

Si l'on oppose ces deux partis sur un axe pro-Québec ou pro-Ottawa, on constate également que les deux sont résolument centrés sur le Québec, exigeant non seulement le respect des juridictions accordées à la province, mais manifestant aussi la volonté d'occuper activement leurs propres champs de compétence tant dans les secteurs politique et culturel que dans les domaines économique et social.

Ainsi, lorsque l'on considère globalement l'ensemble de la période 1960-1968, le Parti libéral peut être considéré comme plus interventionniste que l'Union na-

20 Discours prononcé le 24 mai 1963 à l'Université Western Ontario et reproduit en partie dans jean Lesage, op. cit., $p .40$.

21 Discours prononcé le 20 septembre 1964 devant la Fédération libérale du Québec et reproduit en partie dans jean Lesage, op. cit., $p$. 29.

22 Daniel Johnson, Égalité ou indépendance, Montréal, Renaissance, 1965, p. 105.

23 Ibid., p. 109. Voir aussi Paul Gros d'Aillon, Daniel Johnson, l'égalité avant l'indépendance, Montréal, Stanké, 1979. 
tionale. En outre, même si ces deux partis proposent des mesures qui présentent une orientation plus individualiste que collectiviste, le Parti libéral apparaît tout de même plus collectiviste que l'Union nationale au cours de cette période, si bien que l'on peut opposer ces deux partis selon cette double polarité. Par contre, ces deux partis tendent de plus en plus à se rapprocher vers la fin de cette période, puisque l'Union nationale devient plus interventionniste et aussi plus collectiviste. En somme, on assiste à une concordance de plus en plus grande entre ces deux partis sur la forme d'organisation étatique de la société québécoise (avec certaines différences dans le domaine économique), si bien que la victoire de l'Union nationale en 1966 n'indique pas une rupture avec la situation antérieure.

Outre cette première polarisation qui tend à s'atténuer vers la fin de cette période, ce qui caractérise aussi le système des partis durant ces années, c'est la présence d'un bipartisme tempéré par l'apparition de nouveaux partis qui ne réussissent pas à s'imposer auprès de l'électorat.

Figure 1

Opposition globale du PLQ et de l'UN sur deux axes, 1960-1968

$\underline{\text { Retour à la table des matières }}$

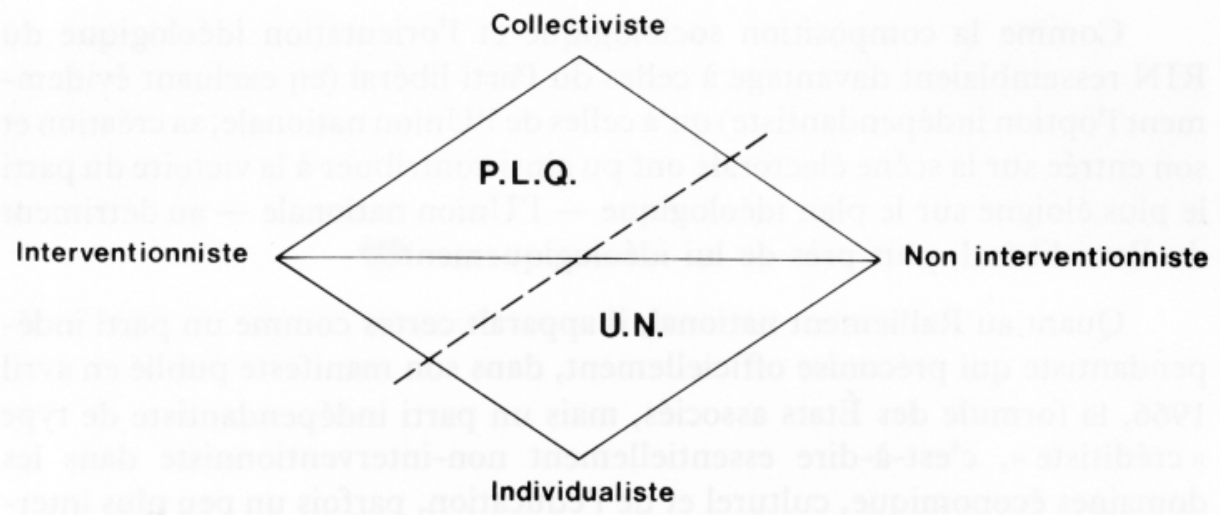

Par suite de la tendance à la convergence du Parti libéral et de l'Union nationale sur l'axe interventionniste, vont se développer de nouvelles tensions centrifuges incarnées dans deux nouveaux partis - le Rassemblement pour l'indépendance 
nationale (RIN) et le Ralliement national (RN) - qui présentent en même temps la caractéristique essentielle d'être indépendantistes.

La transformation du Rassemblement pour l'indépendance nationale en parti politique en mars 1963 et surtout sa présence sur la scène électorale en juin 1966 ne coïncident pas avec des changements importants dans la distribution et la composition de l'électorat. Ainsi, le RIN doit faire appel à la fois à l'électorat nationaliste de l'Union nationale avec un succès plutôt mitigé et surtout à la nouvelle classe moyenne déjà courtisée par le Parti libéral, mais susceptible d'être séduite par un programme qui préconisait non seulement l'indépendance politique du Québec, mais aussi une planification de l'activité économique et des affaires socio-culturelles réalisée sous l'égide de l'État.

La planification économique, dans l'optique du RIN, devait permettre à l'État québécois d'acquérir la maîtrise des facteurs de production et d'agir sur la conjoncture selon les besoins de l'économie. Aussi l'État était-il appelé à intervenir face à l'investissement et à la consommation, à exercer son influence sur le développement de l'industrie et l'aménagement des régions moins développées, à mettre en place des structures pour intégrer les investissements étrangers au plan national de développement et pour rationaliser le commerce extérieur. La planification économique devait aussi s'accompagner d'une planification dans les affaires socio-culturelles, de manière à protéger les citoyens contre les risques normaux de l'existence (accidents, maladie, etc.) et à organiser et démocratiser l'éducation, la culture et les loisirs ${ }^{24}$.

Comme la composition sociologique et l'orientation idéologique du RIN ressemblaient davantage à celles du Parti libéral (en excluant évidemment l'option indépendantiste) qu'à celles de l'Union nationale, sa création et son entrée sur la scène électorale ont pu ainsi contribuer à la victoire du parti le plus éloigné sur le plan idéologique - l'Union nationale - au détriment du Parti libéral, plus près de lui idéologiquement 25.

24 Voir mon article, «L'idéologie du RIN : une idéologie d'affirmation », dans Idéologies au Canada français, 1940-19 75, Québec, PUL (à paraître).

25 Le PLQ et l'UN se ressemblaient au niveau des programmes et des principaux dirigeants, mais non au niveau du membership et de l'électorat. 
Quant au Ralliement national, il apparaît certes comme un parti indépendantiste qui préconise officiellement, dans son manifeste publié en avril 1966, la formule des États associés, mais un parti indépendantiste de type « créditiste », c'està-dire essentiellement non-interventionniste dans les domaines économique, culturel et de l'éducation, parfois un peu plus interventionniste dans le secteur social, et surtout très soucieux de défendre les libertés individuelles face aux envahissements de l'État. «Un gouvernement juste, soutient le parti, doit respecter et assurer la primauté absolue des droits de la personne humaine et de la famille 26 ».

On peut donc déterminer globalement la position des principaux partis politiques oeuvrant sur la scène québécoise au cours de la période 1960-1968 en les rangeant sur l'axe interventionniste 27 . Le rapprochement entre les deux partis dominants sur cet axe à partir de 1965-1966 et l'apparition de deux partis indépendantistes ouvrent la voie à une nouvelle rupture qui se concrétisera vraiment au cours de l'année-charnière 1968.

\section{Figure 2}

Position globale des partis politiques québécois

$\underline{\text { Retour à la table des matières }}$

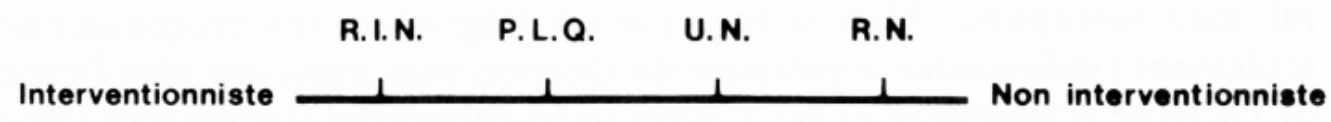

26 « Manifeste du Ralliement national », publié dans La Nation (organe officiel du RN), $2: 1$, avril 1966, p. 5.

27 Il est à noter que ce qui importe dans cette figure 2, c'est la position que les partis occupent les uns par rapport aux autres et non pas leur position exacte sur cet axe. En outre, cette figure indique la dominante qui caractérise globalement le parti pour l'ensemble de la période étudiée. Par exemple, l'UN se montre beaucoup plus interventionniste dans son programme de 1966, mais beaucoup moins en 1960 et en 1962. 


\section{La rupture de 1968}

$\underline{\text { Retour à la table des matières }}$

Tout comme celle de 1960, la rupture de 1968 est avant tout politique ${ }^{28}$. Elle se traduit essentiellement par un fractionnement au sein de la nouvelle classe moyenne entre deux tendances, l'une plus nettement fédéraliste, l'autre plutôt indépendantiste avec toutes les nuances nécessaires à établir dans l'un et l'autre camps. À la limite, on pourrait même parler d'un souverainisme fédéraliste et d'un souverainisme confédéraliste.

Sur le plan politique, on assiste, en effet, à un réalignement des forces avec la naissance du Parti québécois en 1968. Ainsi, une fraction de la nouvelle classe moyenne appuie désormais le Parti libéral, qui préconise un fédéralisme rentable, plus ou moins renouvelé, et une autre fraction appuie le Parti québécois, qui préconise la souveraineté politique du Québec assortie d'une association économique avec le reste du Canada. Il s'agit donc d'une scission avant tout politique au sein de la nouvelle classe moyenne qui soutient, en gros, le même projet néocapitaliste avec certaines variantes, selon que l'on met l'accent plutôt sur l'entreprise privée, avec le Parti libéral, ou plutôt sur l'État et le secteur coopératif, avec le Parti québécois ${ }^{29}$.

Par contre, la nouvelle classe moyenne qui s'est fractionnée entre le Parti libéral et le Parti québécois se trouve confrontée, au cours de cette période, d'une façon fort différente aux trois « institutions » dominantes identifiées plus haut. Dans les domaines de l'éducation et des affaires sociales, l'emprise de l'Église est beaucoup moins forte qu'auparavant et l'État a réussi à s'affirmer et à s'imposer dans ces secteurs par la création des Cégeps en 1967 et de l'Université du Québec en 1968, ce qui vient compléter l'établissement d'un réseau public d'enseignement s'étendant de la maternelle à l'université. Désormais la lutte va porter sur les excès

28 On peut aussi noter que cette ligne de clivage politique recoupe, dans une certaine mesure, une ligne de clivage socio-économique fondé sur l'âge, la scolarité, l'occupation et même l'ethnie.

29 Voir Pierre Fournier, «Les tendances nouvelles du pouvoir économique au Québec », Le Devoir, les 9 et 10 juin 1976. 
ou les erreurs des réformes effectuées, en particulier sur la centralisation et la bureaucratisation excessive du ministère de l'Éducation et sur les réformes pédagogiques.

Le même phénomène se retrouve dans le secteur de la santé, avec quelques années de retard, puisqu'il faudra attendre la publication du Rapport CastonguayNepveu avant de procéder aux dernières réformes. Désormais, dans ces deux secteurs, on ne parle plus d'expansion et de croissance, mais plutôt de rationalisation des dépenses et de restrictions budgétaires.

L'affirmation de la nouvelle classe moyenne contre la bourgeoisie anglophone ne s'est pas tellement manifestée au cours des années soixante, puisque la division institutionnelle du travail entre les deux groupes empêchait toute confrontation directe ${ }^{30}$. D'une part, la nouvelle classe moyenne francophone en pleine expansion pouvait facilement occuper les postes que l'État créait au sein de la fonction publique, dans les secteurs des affaires sociales et de l'éducation, dans les organismes publics et parapublics, ainsi que les postes créés dans le secteur coopératif et dans certaines entreprises privées francophones.

D'autre part, la nouvelle classe moyenne anglophone, enrichie de l'apport des immigrants qualifiés, se retrouvait surtout dans les organisations bureaucratiques privées liées au monde de la finance, du commerce et de l'industrie et fortement dominées par la bourgeoisie anglophone, ou encore elle était employée dans la fonction publique fédérale, qui pratiquait largement une politique d'unilinguisme anglophone.

Le problème surgira plutôt au cours de la période 1968-1976, lorsque l'expansion bureaucratique des secteurs public et parapublic francophones atteindra sa limite de croissance et ne pourra répondre aux attentes suscitées par le développement de l'État québécois et du secteur de l'éducation dans la décennie précédente. Cela engendrera une confrontation plus directe avec la bourgeoisie anglophone qui contrôle le secteur privé de l'économie et suscitera les réclamations d'une politique linguistique qui puisse satisfaire les revendications de la nouvelle classe

30 Hubert Guindon développe ce thème dans « La modernisation du Québec et la légitimité de l'État canadien », Recherches sociographiques, 18 :3, septembredécembre 1977, pp. 337-366. [Texte disponible dans Les Classiques des sciences sociales. JMT.] 
moyenne francophone tant au niveau de la langue de travail que de la langue d'enseignement (des lois 63 et 22 jusqu'à la loi 101).

Enfin, l'affirmation de l'État québécois face à l'État fédéral prendra un sens nouveau après 1968. De 1960 à 1968, il s'agissait avant tout pour l'État québécois de récupérer certains champs de compétence envahis par Ottawa ou, tout au moins, d'occuper pleinement ses propres domaines de juridiction et surtout d'obtenir les pouvoirs fiscaux nécessaires pour faire face à ses obligations. Après 1968, la situation va changer. Avec l'arrivée de Pierre Elliott Trudeau à la tête du pays, le nouveau gouvernement fédéral refuse non seulement d'accorder un statut particulier au Québec, mais aussi de lui reconnaître toute forme de caractère distinctif. Il croit au contraire pouvoir satisfaire les aspirations du Québec par l'élaboration d'une politique de bilinguisme dans les services publics et une présence plus massive des francophones à Ottawa, tant dans la fonction publique que dans les sphères politiques. Cette politique de bilinguisme se révélera à la fois irritante pour un grand nombre d'anglophones et insatisfaisante pour un grand nombre de francophones.

À Québec, durant ce temps, les partis vont se réaligner sur le plan politique et adopter plus résolument la position fédéraliste pour les uns (Parti libéral, Union nationale et même Ralliement créditiste) ou, au contraire, opter pour une forme d'indépendance du Québec (Parti québécois). En 1968, en effet, le Parti libéral se range nettement dans le camp fédéraliste en ignorant la thèse du statut particulier préconisée par Paul Gérin-Lajoie après avoir refusé, l'année précédente, l'option de René Lévesque. Ce qui permet à ce dernier de fonder le Mouvement souveraineté-association en 1967, puis de présider à la naissance du Parti québécois l'année suivante par fusion avec le Ralliement national - le RIN préfère alors se saborder au profit du nouveau parti plutôt que de connaître une mort lente ${ }^{31}$. En 1968 aussi, l'Union nationale perd son chef, Daniel Johnson, qui s'était montré ferme dans ses négociations avec Ottawa et que l'on identifiait encore à l'option « égalité ou indépendance 32 »: il est remplacé par Jean-Jacques Bertrand, qui préfère le compromis à la fermeté et qui se montre plus conciliant dans ses négociations avec le gouvernement d'Ottawa.

31 Le RIN se saborde lors de son congrès des 26 et 27 octobre 1968, alors que le PQ est fondé deux semaines plus tôt.

32 Selon le titre de son volume publié en 1965. 
Ainsi, après la phase de construction et d'organisation de l'État québécois (1960-1968), ce qui engendrait une opposition première entre les partis selon leur conception du rôle de l'État, s'ouvre la période dominée par le problème de la place que doit occuper l'État québécois dans la fédération canadienne, ce qui oppose les partis selon l'axe fédéralisme-indépendantisme. À cette opposition première s'ajoute une seconde ligne de clivage selon le degré d'intervention de l'État dans la société québécoise.

\section{Les partis et le fédéralisme canadien, 1968-1976}

$\underline{\text { Retour à la table des matières }}$

Pour cerner d'une façon plus précise ces lignes de clivage entre les partis politiques québécois, on peut poser le problème de la place de l'État québécois selon une double dimension. D'une part, la place que doit occuper l'État québécois au sein de la fédération canadienne, ce qui engendre une crise de légitimité du système avec polarisation entre fédéralistes et indépendantistes. De l'autre, la place de l'État québécois au sein même du Québec, concrétisée par exemple par la remise en cause du rôle de l'État à la fois par les syndicats et le patronat mais pour des raisons différentes, ce qui engendre une crise de consensus dans le système, avec polarisation entre interventionnistes et non-interventionnistes.

Lorsque le Parti québécois se présente devant l'électorat en 1970, il lui soumet une option nouvelle s'apparentant à celle du RIN mais profondément atténuée sur le plan économique. C'est ainsi que le programme de 1970 apparaît comme primordialement politique (même si ce secteur se retrouve à la fin du programme) puisqu'il s'agit de définir avant tout l'État québécois, de bien préciser sa place sur la scène canadienne, avant d'indiquer ce qu'il fera et comment il le fera. La démarche péquiste de 1970 repose donc sur un postulat très simple : la nécessité préalable de l'indépendance ou, tout au moins, d'un Québec souverain qui a récupère ses pouvoirs et ses impôts, mais qui accepte de partager certaines juridictions économiques. 
C'est cet État à la fois souverain et associe qui pourra assurer le développement d'une société "moderne et originale », tant dans le domaine culturel que dans les secteurs économique et social. Partout est posé le préalable de la souveraineté politique comme condition nécessaire, et parfois presque suffisante, d'un développement culturel conçu et planifié par un État qui tend à coïncider avec la nation, d'un développement social axé sur le travail, la santé, l'habitation et la protection du consommateur, d'un développement économique réalisé avant tout par le secteur public.

Partout, on fait appel à l'intervention de l'État. Ainsi, au chapitre de la vie sociale, tout en réclamant la participation active des citoyens afin d'éviter une bureaucratisation excessive de ce secteur, le programme affirme sans ambages qu'il « faut accepter une fois pour toutes que le rôle primordial, qui est d'élaborer et d'administrer les politiques, soit rempli par l'autorité populaire suprême, l'État 33 ». Le programme est encore plus explicite sur le rôle central dévolu à l'État au chapitre de la vie économique puisqu'il reconnaît l'État comme « le grand moteur des économies contemporaines » et que l'État souverain du Québec a « tout particulièrement le rôle d'élargir la taille de notre économie » et ce, non pas en multipliant les contrôles ou les réglementations applicables aux entreprises privées, mais en créant ses propres instruments d'action ${ }^{34}$. S'appuyant sur ces principes, le Parti québécois en tire une conclusion logique : « ... l'État doit adopter comme forme prioritaire d'intervention dans l'économie une extension soutenue du secteur public (entreprises d'État ou mixtes) 35 ».

Ce rôle central est réaffirmé dans les programmes de 1973 et de 1976 36, bien que l'on en atténue l'approche. Certes, le parti place encore l'État et les entreprises publiques au cœur du développement économique, niais reconnaît le concours du monde du travail et des entreprises privées à l'élaboration d'un plan destiné à moderniser et restructurer l'économie, et surtout accorde désormais au secteur coopératif une place privilégiée dans le fonctionnement de l'économie. Le parti s'engage

33 La solution. Le programme du Parti québécois, Montréal, du Jour, 1970, p. 51. [Texte disponible dans Les Classiques des sciences sociales. JMT.]

34 Ibid., p. 25.

35 Ibid., p. 26.

36 En fait, c'est le programme daté de 1975 que l'on soumet à l'électorat québécois en novembre 1976. 
aussi à favoriser les formes collectives d'organisation et à assurer la participation des travailleurs aux décisions.

Cette esquisse d'une voie québécoise vers la social-démocratie se trouve reprise et complétée au chapitre de la vie sociale, centrée sur une « véritable politique de redistribution et d'utilisation sociale des ressources », afin d'en arriver à « l'établissement d'une justice sociale basée sur une juste répartition de la richesse et sur l'élimination complète de la pauvreté 37 ». Plus encore que le secteur économique, c'est davantage le domaine social qui imprime au programme une orientation social-démocrate. En d'autres termes, le Parti québécois est plus disposé à socialiser le mode de répartition ou de distribution des biens que son mode de production, tendance que l'on retrouve aussi chez les autres partis politiques. Mais le Parti québécois va plus loin que les autres en se disant prêt à « subordonner les critères de rentabilité économique aux critères de rentabilité sociale 38 ».

On pourrait aussi croire que le Parti québécois est désormais bien identifié à l'option souveraineté-association, même s'il a ajouté l'étape importante du référendum dans son programme de 1975 39, et qu'il s'attache de plus en plus à définir le type de société à construire après l'accession à l'indépendance, ce qui est de nature à réconcilier les «électoralistes » et les "participationnistes » au sein du parti. Cette évolution du programme est d'ailleurs confirmée par des données

37 Le programme, l'action politique, les statuts et règlements, Édition 1973, p. 17.

38 Ibid., Édition 1975, p. 11.

39 C'est au cours de la campagne électorale fédérale de 1972 que certains leaders du PQ lancent pour la première fois l'idée d'une consultation populaire sur la question de l'indépendance dans l'éventualité de l'accession au pouvoir du parti. Des annonces, publiées par le PQ lors des élections provinciales de 1973, reprennent cette idée d'un référendum sur l'avenir du Québec. Ce n'est cependant qu'à la suite du congrès de novembre 1974 que va apparaître, dans le programme officiel du PQ, l'engagement de recourir au référendum avant de déclarer unilatéralement l'indépendance du Québec. Cet engagement est modifié à nouveau dans le programme de 1978, alors que le Parti québécois promet de « s'assurer, par voie de référendum et au moment qu'il le jugera opportun, à l'intérieur d'un premier mandat, de l'appui des Québécois sur la souveraineté du Québec ». Enfin, la question soumise à l'électorat québécois lors du référendum de mai 1980 annonce un second référendum à la suite du premier qui ne doit porter que sur l'octroi d'un mandat de négocier une nouvelle entente entre le Québec et le Canada, basée sur l'option de la souveraineté-association. 
quantitatives, puisque l'importance initiale accordée au secteur politique ne se retrouve plus dans les versions ultérieures du programme (1973 et 1975), qui cèdent la place au monde économique et, dans une moindre mesure, à la vie sociale. Il en est de même dans le domaine culturel, où le très grand nombre de propositions de 1970 diminue de moitié dans les programmes suivants.

Bref, le Parti québécois se montre plus habile dans la présentation de son programme. Celui-ci dégage désormais une vision plus globale et moins sectorielle des problèmes de la société et surtout il atténue le dirigisme initial par la recherche de la concertation et le développement de la coopération. Mais la dominante demeure toujours la volonté d'intervention de l'État dans les secteurs politique et socio-économique, accompagnée d'une «libéralisation » du programme dans le secteur culturel après 1970. Ce qui caractérise aussi ce parti, c'est que l'interventionnisme étatique doit se réaliser avant tout au profit de la collectivité - ce qui le distingue à ce niveau du Parti libéral de la Révolution tranquille qui se montrait un peu plus individualiste - bien que les mesures individualistes aient tendance à croître entre 1970 et 1975. Mais la différence la plus sensible entre le Parti québécois et le Parti libéral des années soixante se situe plutôt au niveau politique, où le Parti québécois prône une option souverainiste, ce qui rejoint la rupture de 1968 et peut aussi expliquer sa tendance plus collectiviste.

De son côté, le Parti libéral a choisi clairement l'option fédéraliste dans son programme de 1970 : « ... le Parti libéral opte sans équivoque pour le maintien du régime fédéral dont la souplesse en fait le système le plus efficace et le plus apte à garantir la réalisation des objectifs fondamentaux du Québec ${ }^{40}$. » Mais ce fédéralisme doit tenir compte des particularités du Québec et consentir à un partage nouveau des champs de compétence et des ressources fiscales. Cette profession de foi sera reprise lors des élections de 1973, tout en préconisant un fédéralisme décentralisé qui soit conciliable avec la souveraineté culturelle du Québec. C'est à peu près le même thème qui est évoqué en 1976, bien que cette fois-ci la question constitutionnelle introduise le programme au lieu d'être reléguée dans les dernières pages au profit des autres secteurs d'activité. Il faut aussi souligner que le fédéralisme du Parti libéral est fondé très souvent sur des arguments économiques et nullement identifié à une forme de nationalisme canadien.

40 Québec : Au travail !, programme du PLQ, 1970, p. 52. 
Au total, un fédéralisme sans faille, affirmé clairement dans tous les programmes, requérant il va sans dire certaines modifications, mais s'opposant surtout à un séparatisme qui « condamne le Québec à des retards tragiques sur le plan de son développement 41 ». D'où la polarisation entre une option nettement fédéraliste et l'option de la souveraineté-association et la difficulté pour les autres partis (l'Union nationale et le Ralliement créditiste) de se donner un caractère distinctif sur cet axe fédéralisme-indépendantisme.

Si le Parti libéral se situe nettement au pôle fédéraliste et qu'il réaffirme avec vigueur son attachement au fédéralisme, on constate en même temps que sa conception du rôle de l'État québécois évolue de plus en plus vers la nonintervention entre 1970 et 1976, comme si une position trop nettement fédéraliste entraînait une diminution conséquente du rôle de l'État québécois. En 1970 et 1973, il se montre favorable aux interventions de l'État dans le développement du secteur social et, à l'occasion, de la vie culturelle, mais cet interventionnisme est surtout orienté vers la prestation de biens et de services aux individus. En 1976, par contre, alors qu'il se proclame « le seul parti politique québécois vraiment fédéraliste 42 », il devient en même temps plutôt non-interventionniste, en particulier au chapitre de la vie économique. Globalement, le parti ne cherche nullement à diriger ou à contrôler l'économie québécoise, mais plutôt à l'orienter et à la rationaliser, tout en reconnaissant clairement que l'État « ne peut assumer toutes les responsabilités » et que "l'initiative privée conserve toute son importance dans la vie économique, sociale et culturelle de la collectivité 43 ». Cette orientation est d'autant plus significative que le parti a toujours accordé une importance primordiale à la vie économique au cours de cette période.

On assiste donc de plus en plus à un partage des tâches entre le secteur privé, qui est appelé à assurer le développement économique, et l'État, qui prend en charge le social et, au besoin, le culturel. C'est davantage l'État-bienfaiteur qui domine et non plus l'État-organisateur des années de la Révolution tranquille. Ce même partage des tâches se retrouve aussi au niveau politique, où le parti se présente comme l'unique défenseur de la thèse fédéraliste et laisse au Parti québécois le soin de défendre celle de la souveraineté politique.

41 Un nouveau programme d'action, 1973, p. 75.

42 Programme 1976, p. 3.

43 Ibid., p. 7 (souligné dans le texte). 
Pour sa part, l'Union nationale se montre globalement plus interventionniste que le Parti libéral en 1970 ; elle s'engage à mettre en place les « instruments de la conquête économique » que sont la Société générale de financement et la Société de développement industriel. C'est ainsi que le programme de 1970 s'ouvre sur un acte de foi en l'État du Québec : pour les Québécois, «l'État du Québec est un instrument privilégié pour contrôler et orienter leur devenir collectif. L'État devient un outil essentiel du progrès économique et social de la nation. Dans la mesure même où l'État est perçu comme utile et nécessaire, le Québec lui-même, comme entité nationale, se trouve rehaussé dans le sentiment de ses citoyens ${ }^{44}$. »

Le rôle accordé à l'État dans la vie économique et sociale rapproche davantage l'Union nationale du Parti québécois, alors que le Parti libéral et le Ralliement créditiste tendent à se rapprocher dans leur conception d'un État qui laisse une large place à l'entreprise privée, comme en témoigne cette prise de position du Ralliement créditiste : « Nous croyons que la production des biens de consommation et des services, pour des raisons d'efficacité, doit être assurée par l'entreprise privée. À ce chapitre le rôle de l'État doit se limiter à la protection des consommateurs et à veiller pour que jouent les mécanismes de la concurrence. l'État se doit d'intervenir au niveau de la distribution des richesses de façon à assurer le bienêtre des couches défavorisées de la société 45 . » Cette conception est conforme à la philosophie créditiste basée sur le respect de l'individu et des libertés individuelles et favorable au développement de l'entreprise privée, tout en s'opposant aux abus du système et, en particulier, à la création des monopoles, « qui sont la négation de la liberté économique 46 ». Le Parti libéral, dans son programme de 1970, se montre lui aussi favorable à l'entreprise privée et, à l'occasion, va compter sur l'action de l'État pour assurer le développement économique.

Au total, on peut ranger ces quatre partis politiques sur l'axe interventionniste, comme l'indique la figure 3. La position qui en résulte pour chacun des partis traduit sa conception du rôle de l'État selon le programme de 1970 laquelle recoupe largement la position du parti sur l'axe fédéralisme-indépendantisme. En d'autres termes, plus un parti s'affirme indépendantiste, plus il se veut interventionniste et, à l'inverse, plus il se montre fédéraliste, plus il se définit comme non-

44 Objectifs 1970. Programme de l'Union nationale, préface, p. 3.

45 Camil Samson et le défi créditiste, Québec, Griffon, 1970, p. 125.

46 Ibid., p. 98. 
interventionniste, sans que ceci n'indique nécessairement une relation de cause à effet entre les deux (comme dans le cas du Ralliement créditiste par exemple).

\section{Figure 3}

Position globale des partis politiques québécois sur deux axes en 1970

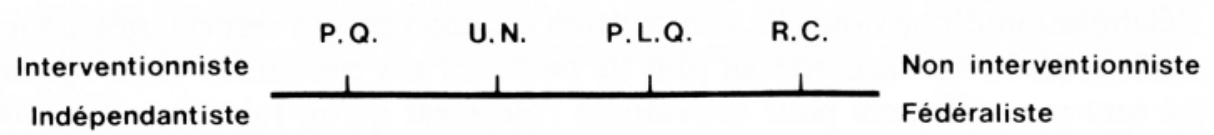

$\underline{\text { Retour à la table des matières }}$

C'est ainsi qu'en 1970, le Ralliement créditiste et le Parti libéral affirment tous deux leur foi dans le fédéralisme comme formule politique tout en exigeant certaines modifications à la Constitution. Le Ralliement créditiste, en outre s'oppose très fortement au Parti québécois, qu'il considère non seulement comme « séparatiste », mais aussi et surtout comme « socialiste 47 ». Ces deux partis, tout en étant les plus fédéralistes, sont aussi les moins interventionnistes. L'Union nationale, pour sa part, veut surtout s'assurer que se réalise la révision de la Constitution, de façon à accorder au Québec « tous les pouvoirs nécessaires à son épanouissement normal et légitime 48 », sans faire référence aux bienfaits du fédéralisme comme formule politique : ce parti se situe, en même temps, plus près du Parti québécois dans sa conception du rôle de l'État.

Cette première forme de réalignement des partis politiques québécois après 1968 va subir quelques modifications par la suite. Du côté indépendantiste, on avait ressenti dès le départ la nécessité d'un regroupement des forces favorables à la souveraineté du Québec dans un seul parti pour que celui-ci soit viable sur la scène québécoise. C'est ainsi que de la fusion du Mouvement souverainetéassociation et du Ralliement national devait naître le Parti québécois et cette création devait entraîner immédiatement le sabordement du Rassemblement pour l'indépendance nationale.

47 Ibid., p. 131.

48 Objectifs 1970. Programme de l'Union nationale, p. 65. 
Du côté des forces fédéralistes, la lutte se fait surtout entre l'Union nationale et le Parti libéral du Québec, puisque le Ralliement créditiste préfère faire appel, dans l'ensemble, à un électorat stable qui lui est acquis sur le plan idéologique plutôt que de risquer de devenir un parti ouvert, plus pragmatique, sans appel idéologique bien défini ${ }^{49}$. Malgré la présence d'Yvon Dupuis à sa tête, le Ralliement créditiste a pu conserver ses appuis électoraux en $1973{ }^{50}$, puis voir ces appuis diminuer de moitié en 1976, alors qu'il maintient sa position noninterventionniste et atténue sa position fédéraliste en se présentant désormais comme le parti de la «troisième voie », soit celle d'un statut spécial pour le Québec, ou comme « la planche de salut 51 », puisque le parti a fait son nid « en plein centre, entre le fédéralisme inconditionnel et le séparatisme suicidaire 52 ».

En 1973, l'Union nationale fera largement les frais de la polarisation qui jouera amplement à la fois au profit du parti dominant chez les forces fédéralistes, le Parti libéral, et au profit de l'unique parti indépendantiste, le Parti québécois. Dans son programme de 1973, en effet, l'Union nationale se rapproche du Parti libéral en se définissant comme moins interventionniste qu'auparavant, en particulier au plan économique, tout en essayant de se démarquer plus nettement de ce parti sur le plan politique en préconisant l'élaboration d'une nouvelle constitution canadienne qui respecterait la formule fédérative et accorderait plus de pouvoirs aux provinces. Cette option ne sera pas suffisante pour convaincre l'électorat qu'on lui offre véritablement une troisième voie entre le fédéralisme du Parti libéral et l'indépendantisme du Parti québécois, et cela se traduira par la déroute quasi complète de l'Union nationale.

En 1976, enfin, l'Union nationale essaiera de se donner, sur l'axe fédéraliste, un caractère distinctif qui soit en même temps susceptible d'attirer une large clientèle électorale en puisant dans les clientèles des partis fédéralistes en place et sur-

49 La tentative d'Yvon Dupuis d'« ouvrir le parti » et de faire appel à un électorat plus urbain s'est avérée un échec désastreux en 1973.

50 Le parti conserve globalement ses appuis électoraux (11\% des voix en 1970 contre $10 \%$ en 1973), mais ces appuis sont désormais plus diversifiés par suite des gains réalisés en milieux urbains qui viennent compenser les pertes subies en milieux ruraux.

51 C'est là le titre de deux brochures publiées par le parti en 1976.

52 Camil Samson, Laurent Legault, J.-Noël Gravel, La planche de salut, 1976, p. 60. 
tout dans celle du Parti libéral. Cette fois-ci, la démarche est apparue plus crédible et l'Union nationale a pu contribuer à la défaite du parti dominant parmi les forces fédéralistes au profit de l'unique parti indépendantiste.

On assiste donc, au cours de la période 1968-1976, à une polarisation de plus en plus forte entre les partis politiques sur l'axe fédéralisme-indépendantisme, polarisation qui favorisera les partis présentant la position plus « extrême », au détriment des partis cherchant une «troisième voie » entre ces deux options. En même temps va se réaliser un glissement de tous les partis sur l'axe interventionniste alors que le Parti québécois abandonne certaines formes d'intervention collectiviste et que toutes les forces fédéralistes se montrent de moins en moins interventionnistes quand elles n'optent pas franchement pour la non-intervention.

\section{CONCLUSION}

$\underline{\text { Retour à la table des matières }}$

Dans une perspective canadienne, les tensions centrifuges de 1968-1976 sont beaucoup plus importantes que celles de 1960-1968, qui portent sur la dimension interventionniste, d'autant plus que la distribution des partis sur ce dernier axe au cours de cette période tendait à un rapprochement vers le centre plutôt que vers les extrêmes. En outre, l'opposition des partis face à l'interventionnisme étatique ne remettait pas fondamentalement en cause le système économique en place, puisque, dans un cadre néo-capitaliste accepté de tous les partis, certains insistaient plutôt sur l'entreprise privée pour assurer le développement économique et d'autres sur l'État, auquel on adjoindra plus tard le secteur coopératif.

De plus, du côté anglophone, on accueillait généralement les années de la Révolution tranquille comme une période de rattrapage pour le Québec, qui lui permettrait précisément d'atteindre « le niveau de développement » politique et économique des autres provinces et, en particulier, de l'Ontario. L'opposition à la bourgeoisie anglophone du Québec ne se développera que plus tard lorsque l'État, le secteur parapublic et le secteur privé francophone ne pourront plus offrir tous les emplois nécessaires aux membres de la nouvelle classe moyenne. 
Même si cette période devait amener le remplacement de l'Église par l'État comme nouvelle institution dominante au Québec, elle ne devait pas conduire à un affrontement sérieux entre les deux (en dépit du « bill 60 » par exemple), ni en conséquence à une polarisation Église-État qui aurait été reprise par les partis en place ou qui aurait engendré un nouveau système de partis. L'affrontement opposa plutôt les partisans d'un interventionnisme étatique aux tenants de la nonintervention, même si cette forme de polarisation devait entraîner inévitablement dans le Québec d'alors une opposition à l'Église. C'est en quelque sorte le schéma social du nationalisme ${ }^{53}$ qui domine au cours de cette période.

Par contre, le renforcement de l'État québécois devait conduire assez rapidement durant ces années à un affrontement avec l'État fédéral, en particulier en ce qui concerne des sources accrues de revenu pour le Québec et même une nouvelle forme de partage des pouvoirs ${ }^{54}$, mais sans remettre fondamentalement en cause le cadre fédéral lui-même ou la formule fédérative.

En somme, la période 1960-1968 ne provoque pas une crise de légitimité ou une crise de système, ce qui va caractériser la période suivante (1968-1976), mais plutôt une crise dans le système ou une crise de consensus qui mettra fin à l'unanimité artificielle et apparente des années antérieures sous une idéologie dominante qui laissait croire en une possession tranquille et éternelle de la vérité. Il faut donc situer cette période d'abord et avant tout dans une perspective québécoise, même si les effets de cette mutation rapide se sont aussi faits sentir sur le plan canadien.

La période qui s'étend de 1968 à 1976 a un impact canadien beaucoup plus important, puisque c'est alors le schéma national du nationalisme qui en vient à dominer en opposant les partis selon une dimension fédéraliste et indépendantiste. Désormais, il s'agit de définir de plus en plus la place que doit occuper l'État québécois dans la fédération canadienne, surtout avec l'arrivée du Parti québécois sur

53 Léon Dion établit une distinction entre le schéma national et le schéma social du nationalisme dans Nationalismes et politique au Québec, Montréal, Hurtubise HMH, coll. «Sciences de l'homme et humanisme », no 7, 1975, p. 130. [Livre disponible dans Les Classiques des sciences sociales. JMT.]

54 Comme par exemple la querelle sur l'établissement d'un Régime des rentes du Québec. 
la scène politique, ce qui va conduire à une crise de légitimité du système politique canadien.

En même temps, c'est le Parti québécois qui va reprendre du Parti libéral le flambeau de l'interventionnisme étatique en lui imprimant par contre une orientation plus nettement collectiviste qui vient ainsi rejoindre le sens de la communauté que l'on retrouvait surtout dans le Québec d'autrefois et que l'on cherche désormais à adapter à une situation nouvelle.

Mais au moment où la question nationale polarise le débat entre fédéralistes et indépendantistes et où les partis ont en quelque sorte secondarisé la question sociale, les conflits sociaux surgissent de toutes parts et deviennent de plus en plus aigus (crise d'octobre 70, conflit de La Presse en 1971, front commun de 1972, etc.), de sorte que les partis sont incapables de répondre adéquatement à la situation. Ce sont alors les groupes sociaux qui vont intervenir et s'opposer selon leur conception du rôle de l'État (les centrales syndicales versus le Conseil du patronat par exemple) et selon la forme de développement de la société québécoise 55 . Cela permet ainsi de mieux saisir et de mieux comprendre l'ampleur et l'acuité des crises sociales sous le gouvernement Bourassa.

On constate donc qu'à chacune des époques, le social et le national sont présents et dominent tour à tour, mais c'est avant tout la dialectisation constante du national et du social qui se trouve à la base de la dynamique sociétale et partisane au Québec au cours de l'ensemble de la période 1960-1976. En outre, si c'est vraiment le schéma national du nationalisme qui domine au cours de la période 1968-1976, il faut alors en conclure que l'arrivée au pouvoir du Parti québécois, le 15 novembre 1976, ne marque pas une rupture avec la période antérieure, mais plutôt une continuité ou, plus exactement, l'aboutissement normal et logique de cette période. En d'autres termes, la question nationale polarise toujours les partis politiques québécois et dominera la scène québécoise au moins jusqu'à l'après référendum, de sorte que la question sociale est à nouveau secondarisée par rapport à ce problème plus urgent, bien que de plus en plus l'une et l'autre tendent à s'interpénétrer. Ainsi, le néo-nationalisme, défini et imposé par la nouvelle classe moyenne au pouvoir, est devenu la nouvelle idéologie d'unification de la société

55 Voir à ce sujet l'article suivant de Raymond Hudon sur « Les groupes et l'État québécois ». 
québécoise, surtout dans sa composante étatiste, qui cherche à transcender ou à occulter les divisions au sein de la société et, en particulier, les divisions de classes.

Cependant, à mesure que s'accentue l'emprise de l'État sur l'ensemble des domaines de la vie économico-sociale, s'accentue aussi la contestation de l'État dans tous les secteurs d'activité. On remet en cause le savoir et le pouvoir de la technocratie, la tendance à la centralisation et à la bureaucratisation, son inefficacité économique, en même temps que sont de plus en plus valorisées la participation des citoyens, la décentralisation au niveau local ou régional et la libre entreprise.

Cette contestation est aussi reprise par les partis politiques québécois qui, au cours de la dernière décennie, sont devenus de moins en moins interventionnistes. Mais, pour le moment, c'est encore l'opposition entre fédéralistes et indépendantistes qui domine le débat. Selon les résultats du référendum du printemps 1980 et des élections générales qui suivront, on pourrait assister à un nouveau réalignement des partis politiques à la fois sur l'axe interventionniste et sur l'axe fédéraliste. Le Parti québécois, en atténuant ses positions souverainiste et interventionniste, occuperait la place de l'ancien Parti libéral des années de la Révolution tranquille et le Parti libéral de Claude Ryan, défendant « le Québec des libertés 56 », se montrant moins interventionniste et cherchant à se définir par rapport au nationalisme québécois en préconisant un fédéralisme largement renouvelé, pourrait remplacer l'ancienne Union nationale. Il resterait alors place pour un regroupement de l'Union nationale et du Ralliement créditiste dans un « parti de droite », qui devrait se définir comme fédéraliste (mais là aussi profondément renouvelé) et surtout non interventionniste - a moins que le Parti libéral n'occupe toute cette place - et pour un nouveau Rassemblement pour l'indépendance nationale nettement indépendantiste et interventionniste, ces deux forces politiques acceptant de jouer le rôle de tiers partis aux extrêmes par rapport aux positions plus centristes des deux partis les plus importants.

\section{Fin du texte}

56 C'est là le titre du volume publié par le PLQ et présentant le texte des allocutions prononcées au Congrès d'orientation du parti, du 18 au 20 novembre 1977. 